\title{
An Methodology for Dimension Parameter transfer to Support Variant Product
}

\author{
Weiping Shao*, Yongping Hao, Pengfei Zeng, Chunyan Wang \\ $R \& D$ Center of CAD/CAM Technology, Shenyang Ligong University, \\ Shenyang 110159, China \\ *Corresponding author's Email: shaoweiping3008@sohu.com
}

\begin{abstract}
In variant design of mass customization, sometimes it's necessary to modify some dimensions of product modules. This paper introduces a methodology for dimension parameter transfer related to variant design that based on constraint satisfaction priority of part and assembly connection. In order to research parameter transfer path and build the parameter transfer structure, parts and its variant parameters are classified. Parameter association relations between parts are analyzed based on assembly connection in product. Parameter constraint relations also presented for variant design. And, parameter transfer structure and model is put forward. Constraint forms between dimension parameter are listed and described, and then a reconstructed network structure for dimension constraints relations is built in which every edge has only one direction with purpose of making the network simple and easy to solve the constraints. Finally, using Pro-E as a 3D environment and $\mathrm{VC}++$ as a development tool, all methods are applied to a shaft-hub link of speed reducer product, thus verifying the proposed method.
\end{abstract}

Keywords: variant design; dimension parameter transfer; constraint satisfaction priority; assembly connection

\section{Introduction}

Manufacturing companies have devoted much attention to the contemporary battlefield called mass customization, which aims to satisfy individual customer needs by introducing product proliferation while taking advantage of mass production efficiency [1]. In order to accompany the increasing variety of customers' demands, manufacturers tend to produce different models of the same product by introducing variant design concepts that improve the flexibility of market requirement $[2,3]$. Through change the partial structure modality and parameter of existing product without influence its working principle and basic functional defines, Variant design can satisfy the specific function requirements or customer individual demands to respond market requirement quickly on product design technique. Product variant includes geometry parameter variant and structure configuration variant. Geometry parameter variant generally causes part shape di- mension or assembly dimension to change and it does not influence the product structure. Structure configuration variant selects and configures part sorts or part quantity according to customer individual demands. It usually results in the local structural transformation of the product.

As assembly connections [4] between parts exist in product, some changed part parameters or dimensions are likely to cause the other parameters of the part itself or the other part's parameters to change. Those changed parameters will affect the rest parts and transfer them in the whole product family. When a parameter of certain part is changed according to customer or technical requirement, the other correlative parameters must be modified simultaneously. This problem is very urgent and necessary to resolve. So, researching the parameter sort and constraint relation between dimension parameters of variant product has very important significance and it is the foundation to 
research dimension transfer path and transfer method deeply.

At the present time, correlative research literature about dimension parameter modification and parameter transfer of parts is infrequent. F. Y. Liu [5] has put forward an approach about parameter transfer based on complexity network theory. S. L. Fang $[6,7,8]$ has studied the parameter transfer structure of variant product. Q. M. Wu [9] has discussed the parameter transfer method for product variant design based on three-dimensional model and finite element model. They have resolved some problems on parameter transfer. However, some new methods and theories are needed to solve parameter transfer and constraint relation problems.

In this paper, we introduce a methodology for dimension parameter transfer to support variant design that integrates parameter analyzing and design techniques with complex networks. Part classification is defined and dimension parameter is analyzed. We discuss the constraint relation between parameters and create a corresponding product family parameter transfer model based on analyzing the assembly connections existing among parts and constraint satisfaction priority of part. Finally, we present a case study using shaft-hub link of speed reducer product to verify the method.

\section{Definitions and Division of Parts Classifi- cation}

Several steps [5], including $\mathrm{ABC}$ analysis, name analysis, classification feature extraction of parts, determination of classes and class hierarchy, construction of multiple-classification tree, are mentioned in general classification of the parts manufacturing-oriented. And many kinds of classification methods for parts exist. For example, part can be classified into shaft, plate, cover, box and fork of parts et al in processing sector. The classification is based on product attributes such as product type, dimension, purpose, et al with the purpose of facilitating customers to choose in sale department. The classification can also be divided into standard parts (in accordance with the function, the standard parts can be divided into fasteners, seals and linkers, et al.), outsourcing parts, spare parts, custom-built parts, home-made parts, et al. What is more, parts can be divided into ring, sets, pin, shaft, tube, ring, shell, body, rod, plate, block, blocks, frames, plates, gears, shaped pieces and special pieces, et al in accordance with geometric structure. Various classification methods of parts in differ- ent application areas and environments, and building an effective classification method which is suit for dimension parameter transfer is the basis of researching on the dimension parameter transfer among parts. As a result, combined with features of variant design and analysis of the $\mathrm{ABC}$ for all parts, in this paper, part classification for dimension parameter transfer, which is shown in Table 1, is based on combination functions and group technique.

Standard parts, accounting for $20 \%-25 \%$ of total parts, have simple structure. They have international or national standards of there own. These parts with fixed part shape dimension and parameter series which are not needed to be redesigned and efficiency of product design is to be improved. Parametric templates modeling method based on Tabular layouts of article characteristic is commonly to be used for the standard parts.

Similar parts with moderately complex have large quantities in mechanical products, and these similar parts mainly have the same function but have a slightly difference in shapes and dimensions. Rate of reuse the part about $70 \%$ of the total number is the highest in variant design. For the similar parts design, we can firstly retrieve the similar ones and then modify little of the retrieved one to ultimately get a new part which meets the design requirements, which can avoid a lot of routine tasks and improve the design efficiency, enabling rapid response to market demand. As the similar parts for the structure and shape of a small difference, parametric template modeling method is used for part of gearwheel and et al. Furthermore, modeling method based on parametric feature is used for part of shaft parts and et al.

Complex parts, only accounting for 5\%-10\% of the total parts, have complex structure, strong specific and low rate of reuse. Parametric feature modeling method is usually used for these parts which have not fixed structures and shapes. And the parametric feature modeling method is generally used to create part models with not fixed structures and variant dimension parameters.

In this paper, different modeling methods for parts are utilized according to different types of parts.

The dimensions of parts with different types are certainly not the same and the corresponding variant strategies and methods are not the same. For example, for standard parts and all-purpose parts in variant design, their dimensions can not be changed at will; otherwise the variant result does not belong to the standard parts. As a result, redesigning parts can greatly increase the 
Table 1 Part classification based on parameter transfer

\begin{tabular}{|c|c|c|c|c|c|}
\hline $\begin{array}{c}\text { Part } \\
\text { classification }\end{array}$ & $\begin{array}{c}\text { Complexity } \\
\text { of part } \\
\text { structure }\end{array}$ & $\begin{array}{c}\text { Rates of } \\
\text { reuse }\end{array}$ & $\begin{array}{c}\text { Priority for } \\
\text { parameter } \\
\text { transfer }\end{array}$ & Modeling method & Parts instance \\
\hline Standard parts & simple & high & highest & $\begin{array}{c}\text { SML-based parametric } \\
\text { templates modeling }\end{array}$ & $\begin{array}{c}\text { keys, bolts, } \\
\text { couplings, et al. }\end{array}$ \\
\hline Similar parts & $\begin{array}{c}\text { moderately } \\
\text { complex }\end{array}$ & high & $\begin{array}{c}\text { the second } \\
\text { highest }\end{array}$ & $\begin{array}{c}\text { Parametric template modeling } \\
\text { or feature modeling }\end{array}$ & shaft, gear, et al. \\
\hline Complex parts & complex & low & lower & Parmetric feature modeling & box, turbine blades \\
\hline
\end{tabular}

cost of variant design and can reduce the efficiency of design process at the same time. Generally, standard parts are a series of specifications parts; they can also be changed in the variant design, but they should belong to the standard parts even after changes with different specifications. Variant design for standard parts mentioned in this paper can significantly accelerate the product development process; can reduce manufacturing time and costs. Furthermore, it can respond quickly to customer needs and reduce product costs. Similarly, for other types of parts, appropriate design strategies and methods need to be adopted.

\section{Variant design parameter analyzing}

In machine product, there exists some geometry dimension and shape constraint relations on parts' fit surface on account of joint and assembly connections among parts. When changing a parameter value of certain part, it will bring the dimension parameters of the interrelated part, which fit with it, to change and will transfer the changed parameter value to the other parts in all the product family and lead them to change their parameter value. Therefore, it is important to analyze the parameter sorts and constraint relations of parts.

Parameter analyzing includes product-level parameter analyzing, part-level parameter analyzing and parameter association analyzing. The purpose of productlevel parameter analyzing is mainly to research the primary performance parameters which influence the whole product functions and structures. The aim of part-level parameter analyzing is chiefly to classify the part parameters and extract various part parameters which can drive the structure to change according to requirements. The intention of parameter association analyzing is to build various association relations among parameters that include association parameter between parts and association parameter in part itself. Only by establishing the correct parameter association, can structure changing driven by variant parameter be transferred to the related part and bring the part's structure to change.

\section{A. Product-level parameter analyzing}

In variant product, the product-level parameters are mostly technique parameters. Take water pump as an example, fluid flux, pressure, piston diameter, piston travel and the maximal temperature of pump etc. are all product-level parameters of water pump. Usually, these parameters are chosen by customer according to the product function requirement in variant design. And designer configures the product instance according to customer demands. Product-level parameters mainly influence the structure configuration variant and usually do not participate in parameter transfer directly.

\section{B. Part-level parameter analyzing}

Part dimension parameters have manifold sorts. Usually, dimension parameters can be divided into three sorts: invariable parameter, variable parameter and educed parameter. Take gear in speed reducer as an example, as shown in Figure 1. Invariable parameter keeps unchanged all the time in part variant design. It does not beget the other parameter to change and is not restricted by other parameters, such as parameter $\theta$ and $n$ shown in Figure 1. Variable parameters are those parameters which always can be changed according to requirements in various part of variant design, such as parameter $D_{A}, D_{4}$ and $B$ in Figure 1. They are master parameters which drive the part to change. And the educed parameter is figured out by the other parameters. For instance, parameter $D_{3}=$ $1.6 D_{4}, D_{0}=D_{A}-(10 \sim 14) m$ ( $m$ is modulus of gear), $D_{1}=\left(D_{0}+D_{3}\right) / 2, D_{2}=0.3\left(D_{0}-D_{3}\right), C=(0.2 \sim$ $0.3) B, R \approx 0.5 C$, etc. These parameters' values are all determined by other parameters and they are changed with them. Parameter classification follows the following principles, namely, the structure dimension parameter, or total parameter, which associates with multiparts, such as parameter $d_{4}$, and local parameter, which only determines the structure of part itself, like parameter $B$; independent parameter do not lead the other part parameter to change, so it is called irrelative pa- 


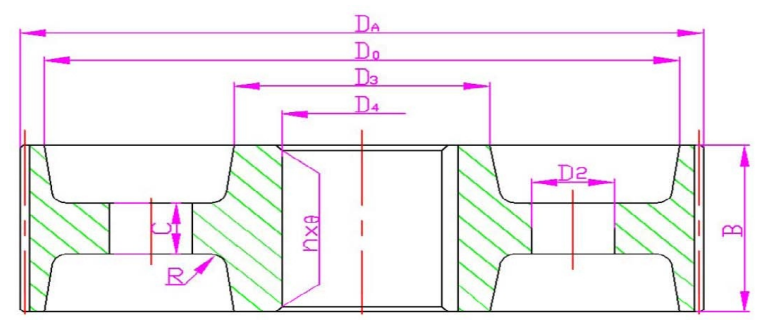

Figure 1 The parameters of gear in speed reducer

rameter, such as parameter $h$.

\section{Parameter association analyzing}

Each part may have one or multi assembly connections with other parts in assembly product. And the portion in part which participates in assembly is called fitting dimension parameter. Parameter association of parts indicates that fitting dimensions have the reciprocity relations. So, parameter association relation is created according to the fitting dimension parameters in product.

\section{Assembly connection analyzing}

Assembly connections among parts in assembly model reflect the geometry constraints of parameters and influence parameter transfer path. Geometry constraint relation among parts, including geometry shape and geometry dimension constraint relation, is determined in the light of assembly connection among parts. Constraint between parts is influencing each other. Some geometry constraints only act on the two correlative parts and do not affect the other parts. Some geometry constraints not only restrict the two correlative parts but also influence the other parts, namely, constraint has transferability and this kind of constraint is called total constraint. Geometry constraint transfer depends on variable parameters and parameter association. Variable parameters are transferred within part or to the correlative parts because of assembly connections existing in product.

There are many kinds of assembly connections in mechanism product. They are mainly separated into four sorts, as shown in Figure 2, including location connection, link connection, motion connection and fit connection.

- Location connection is used to describe the relative location connection between two parts of product, such as coaxes, insert, and tangent, align etc. Interspaces location connection and fit connection among machine parts can be expressed by defining these connections between parts.

- Link connection is mainly used to describe the link mode between assembly parts, including screw thread link, key link, pin link, jointing etc. Link connection can also be divided into knock-down link and unknock-down link. And some of link connections can be subdivided into multifold sorts too. For example, pin link can be sub-divided into cylindrical pin link and taper pin link.

- Motion connection is mainly used to describe the motion mode and drive connection. For instance, crank whirligig, gear drive, chain transmission, etc.

- Fit connection is used to describe the fit compactness degree between parts, such as clearance fit, transition fit and interference fit.

According to analysis the existing kinds of assembly connection, parts with assembly relations have a directly or indirectly relevance relationship. Parts with a typical relationship can be viewed as an assembly object (sub-assembly). Transfer direction of dimension parameter for the sub-assembly is to be determined according to part-types and then constraint satisfaction priority of the parts is provided. In the design process, parameter-associated design method is applied. Dimensions relations and constraints analysis processes can be greatly simplified, which can simplify the relationships among dimensions constraints, and cut down path of dimensions transfers too.

There are two ways of sub-assembly identification: human-computer interaction and automatic identification. Firstly, some sub-assembly or components are determined according to product functions. For example, cover and seal to be seen as a component. Then, other sub-assembly is got automatically with assembly relationships and related map information and assembly characteristics of certain parts. Finally, subassembly is viewed as an assembly object, and it will be combined into a node in network of dimension relationships.

Sub-assembly has certain relations with types of mechanical parts, for example, five-kind parts of disk, shaft, box, fork and set. From the point of formation of sub-assembly, the most likely formation is a subshaft assembly, followed by the cabinet, and many other parts attached to the two types. Also, sub-assembly formed by thread connection can become parts combination with stable structures. In the network of dimension relationships, these parts have a distinguishing characteristic, which is they are located in the center of certain areas.

Thus, sub-assembly with stable structures to be encapsulated together is considered as a whole when analyzing parameter transfer of part demission. So, the 


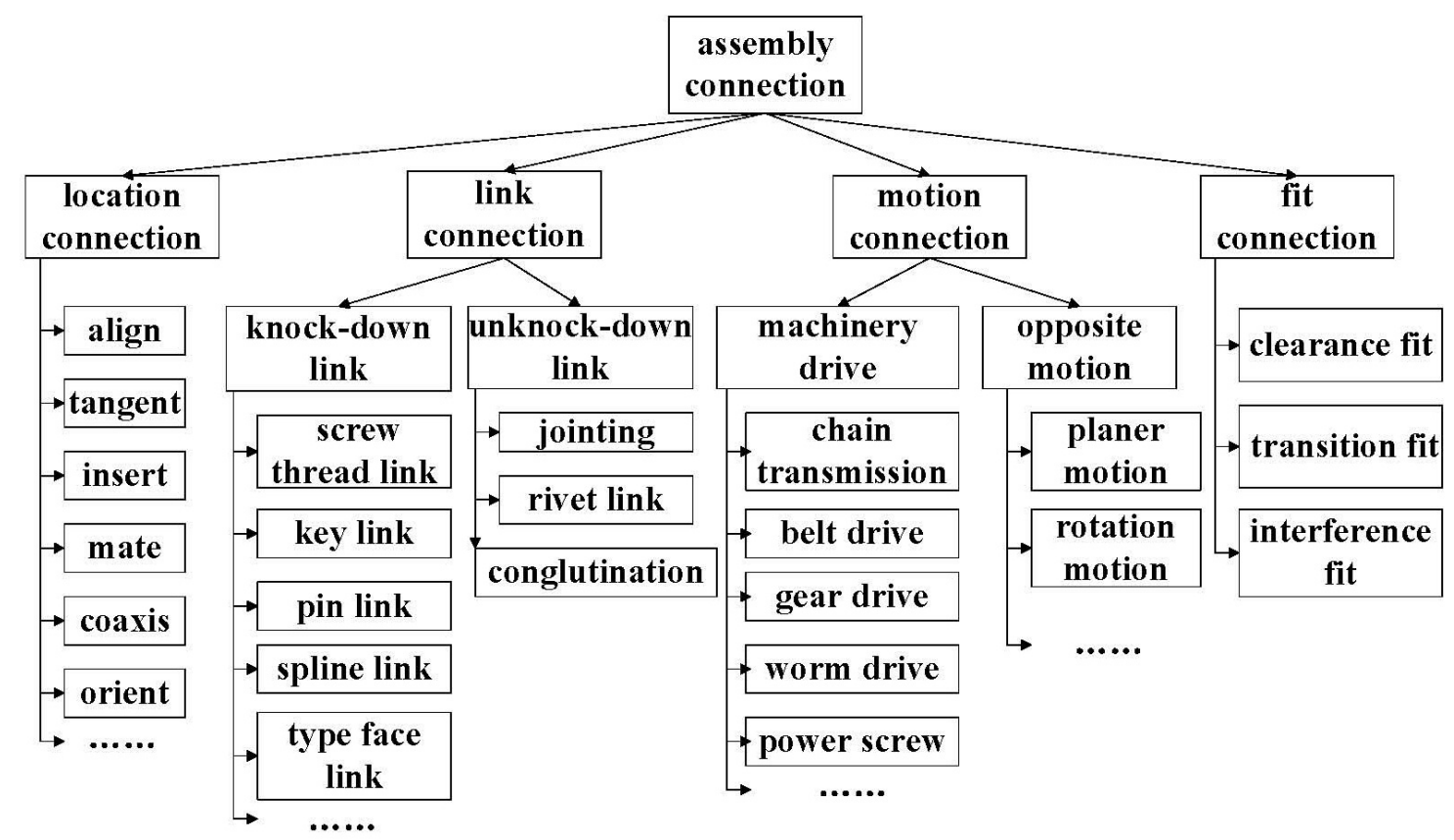

Figure 2 An example of key link

structure of dimension parameter transfer is not only greatly simplified, but also conducive to the transfer of dimensions. And this kind of sub-assembly has characters of specific functions modules, parameters of the structural model and features of interface.

\section{Parameter constraint relation}

Constraint relations between parts indicate the design relation of feature or part. It is used to define the relation expression between dimension, and it is also a sort of mode to capture the design knowledge and intention. Parameter constraint relation is used to drive model design like parameter. Based on forenamed assembly connection sorts of parts, we can see, these parts which compose the assembly connection can be regarded as a subassembly. There exist steady association relations between parts in subassembly interior. Moreover, these association relations are built by using parameter constraint of assembly connection. For example, the assembly connection of key link is made up of shaft, key and wheel hub, as shown in Figure 3.

Take shaft as design benchmark in subassembly of key link. And then, we adopt association design to design the key part and the wheel hub aperture of gear. In this way, when shaft diameter or shape and dimen-

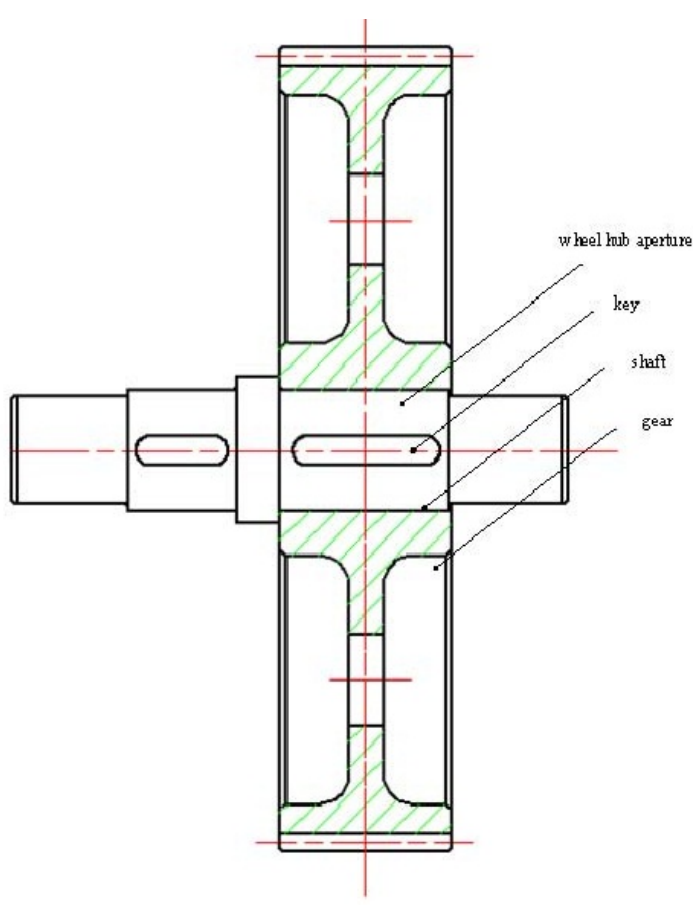

Figure 3 An example of key link sion of the key slot on shaft is changed, the key, the wheel hub aperture and the shape and dimension of key slot of gear which assemble to shaft are all changed. 
The shape and dimension of key are associated with the key slot of shaft. Then the key slot width and length of key slot on shaft are equal to the key width and length respectively. If the key slot structure of shaft is modified, the key structure will be changed with it. Once the key slot structure is determined, the dimension of key is determined too.

It must ensure that the key slot's underside, two side faces and semi-circle face match the corresponding faces of the key when shaft and key fit together. It includes the following constraints: the key's underside matches the key slot's underside as no clearance fit. The key's side face also matches the key slot's side face as no clearance fit. The key's semi-circle is tangent with the key slot's semi-circle.

When key assembles with gear correctly, it must ensure that the shaft axes and the wheel hub aperture axes are coaxes. The key on shaft must match the key slot on gear correctly. On account of the two side faces of key are working faces, they have assembly constraint with the two side faces of wheel hub aperture on gear. Set the fit offset zero. The upside faces of key matches to the underside of wheel hub aperture on gear. There exists a less clearance between them, so set a right fit offset when add constraint.

From the above procedure, we can extract the fit dimension between key and shaft, key and gear, shaft and gear. And then the parameter constraint relation among parts based on it is created. Parameter constraint relation of key link is as shown in Figure 4.

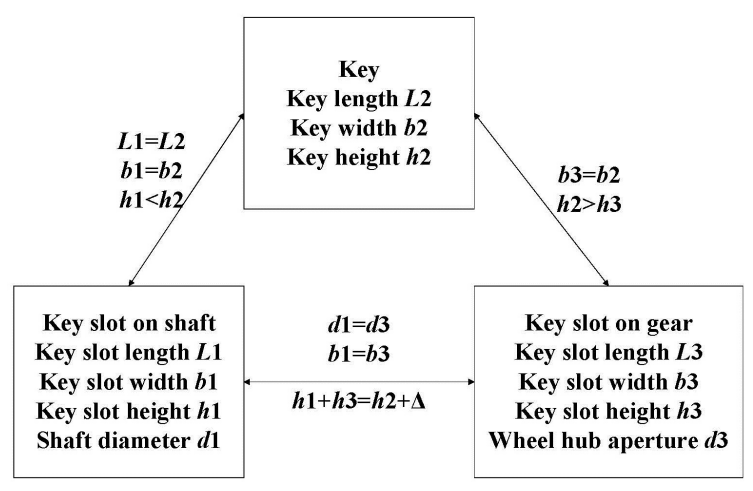

Figure 4 Parameter constraint relation of key link

When designing the key connection, we make the shaft as the basis; associated design based on assembly relations will be carried out for designing gear wheel bore and flat key, so that the assembly key, the gear wheel bore and their shapes and dimensions are changed along with the changing of shapes and dimensions of the shaft diameter and the key slot. De- tailed associated design methods are shown in literature [10].

Define a correlation matrix for parts. The elements (denoted as $S g n_{i j}$ ) in the correlation matrix (Table 2) are assigned following a specific rule. That is, $S g n_{i j}$ is assigned to 1 if parameter(s) from the part $i$ has some associated relations with parameter(s) from the part $j$; otherwise it is assigned to 0 .

\begin{tabular}{c|cccccc}
\multicolumn{7}{c}{ Table 2 The correlation matrix } \\
$P_{i} \backslash P_{j}$ & 1 & 2 & 3 & 4 & $\cdots$ & $\mathrm{m}$ \\
\hline 1 & $S_{11}$ & $S_{12}$ & $S_{13}$ & $S_{14}$ & $\cdots$ & $S_{1 m}$ \\
2 & $S_{21}$ & $S_{22}$ & $S_{23}$ & $S_{24}$ & $\cdots$ & $S_{2 m}$ \\
3 & $S_{31}$ & $S_{32}$ & $S_{33}$ & $S_{34}$ & $\cdots$ & $S_{3 m}$ \\
$\cdots$ & $\cdots$ & $\cdots$ & $\cdots$ & $\cdots$ & $\cdots$ & $\cdots$ \\
$\mathrm{n}$ & $S_{n 1}$ & $S_{n 2}$ & $S_{n 3}$ & $S_{n 4}$ & $\cdots$ & $S_{n m}$
\end{tabular}

Construction of the correlation matrix with the definition is shown in the above table, and its correlation matrix can be found in Table3, Table4, and Table5.

Table 3 Part of a correlation matrix

\begin{tabular}{c|ccc}
$P_{i} \backslash P_{j}$ & $L_{2}$ & $b_{2}$ & $h_{2}$ \\
\hline$L_{1}$ & 1 & 0 & 0 \\
$b_{1}$ & 0 & 1 & 0 \\
$h_{1}$ & 0 & 0 & 1 \\
$d_{1}$ & 0 & 1 & 1
\end{tabular}

Table 4 Part of a correlation matrix

\begin{tabular}{c|cccc}
$P_{i} \backslash P_{j}$ & $L_{3}$ & $b_{3}$ & $h_{3}$ & $d_{3}$ \\
\hline$L_{1}$ & 1 & 0 & 0 & 0 \\
$b_{1}$ & 0 & 1 & 0 & 0 \\
$h_{1}$ & 0 & 0 & 1 & 0 \\
$d_{1}$ & 0 & 1 & 1 & 1
\end{tabular}

\section{Parameters transfer model and dimension constraint network structure}

\subsection{Hiberarchy of parameters transfer}

In order to make parameters associated structure better service for variant design, models to be built must meet the following requirements in the processes of product hierarchical modeling. (1) Levels of product structure are re-organization and described with the purpose of making the parameters transmission chain in a top-down way as short as possible, so that it can reduce parameter transfer path and correspond calculation of parameter data in the product structure. (2) Parameters associational should be as independent as 
Table 5 Part of a correlation matrix

\begin{tabular}{c|ccc}
$P_{i} \backslash P_{j}$ & $L_{2}$ & $b_{2}$ & $h_{2}$ \\
\hline$L_{3}$ & 1 & 0 & 0 \\
$b_{3}$ & 0 & 1 & 0 \\
$h_{3}$ & 0 & 0 & 1 \\
$d_{3}$ & 0 & 0 & 0
\end{tabular}

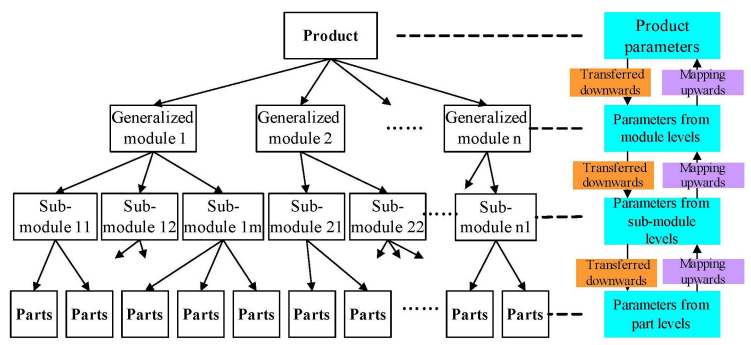

Figure 5 Hiberarchy models for parameter transfer

possible. In the model, only allowing vertical transfer can avoid cross-transfer parameters. The above requirements provide an effective data transfer channels for product variant design. A standardized structure for parameters associated is formed to facilitate analysis of parameter correlation, parameter-driven, and modify the parameters for the future work.

In this paper, product parameters associated structure is built in condition of introducing ideas of parameters design [11] and variable analysis into the modular design combining characteristics of variant design. And we introduce concept of generalized parameters module for building product structure level.

Generalized parameters module [12] is a structure which has specific functions, parametric model, and interface characteristics, and the module is the function of engineering constraints including functional, geometric topology, structural parameters, excitation, and response.

Generalized parameters module is parameterized. Its corresponding interface is parameterized too. Product structure level described using generalized parameters module has broadened the scope of product variant design. The generalized parameters module is referred as generalized module with the purpose of facilitating description in the paper. The generalized module is further divided into several generalized sub-modules according to assembly relation and its features, and the generalized sub-modules consist of parts which are shown in Figure 5.

Through re-organization based on the generalized module, levels of product are significantly reduced; furthermore, path of the top-down variant parameters transferring is shortened which is beneficial for the transfer of variant parameters.

In dimension parameter transfer structures, two kinds of transfer forms, which are transferred downwards and mapping upwards, are used in the paper shown in Figure 5. We can see clearly from Figure 5 that modeling process and variant process has contained. The modeling process is for achieving mapping upwards from part level parameters to product level parameters, and the variant process is for achieving transferred downwards from top level parameters to part level parameters. For horizontal transitive relation of dimensions parameter between parts, we can use parameters associated technique to resolve via assembly relationship, implementation the linkage changes of parameters. Thus only allowing vertical transmission parameters can reduce the size of the complexity of parameter.

Each model module, such as part level, sub-module level, module level and product level, has interface characteristics of the parametric model. And interface parameters, model-driven parameters and model data processing information have contained in their models.

In a modeling process for product variant design, we take parts modeling as a start. Works of setting up part geometry model, analyzing the types of dimension parameters, defining parameters relationships, and determining drive parameters in variant design have finished by using parametric modeling and variation analysis which are based on the tabular layouts of article characteristic or part feature parameters. Parameters Relationships among parts can be built. A parameter can only affect the parameters of part itself which is called a local parameter. While a parameter can not only affect the parameters of the part but also may cause the parameters of other part which is called a global parameter. When dealing with a global parameter we should extract it firstly, and then make it map upwards to its upper sub-module level.

Parameters constraints between relevant parts must be from part driven parameters to its upper sub-model firstly, so it will become a variable of the upper submodel, and then it will be transferred downwards to the parameter of related parts. Part parameters from different sub-modules follow the same associated method that is transferred downwards or mapping upwards.

According to the characteristics of parameter transfer in generalized parameters modules, main variants parameters interrelated are not allowed. All parameters from different parts can not have related relations 


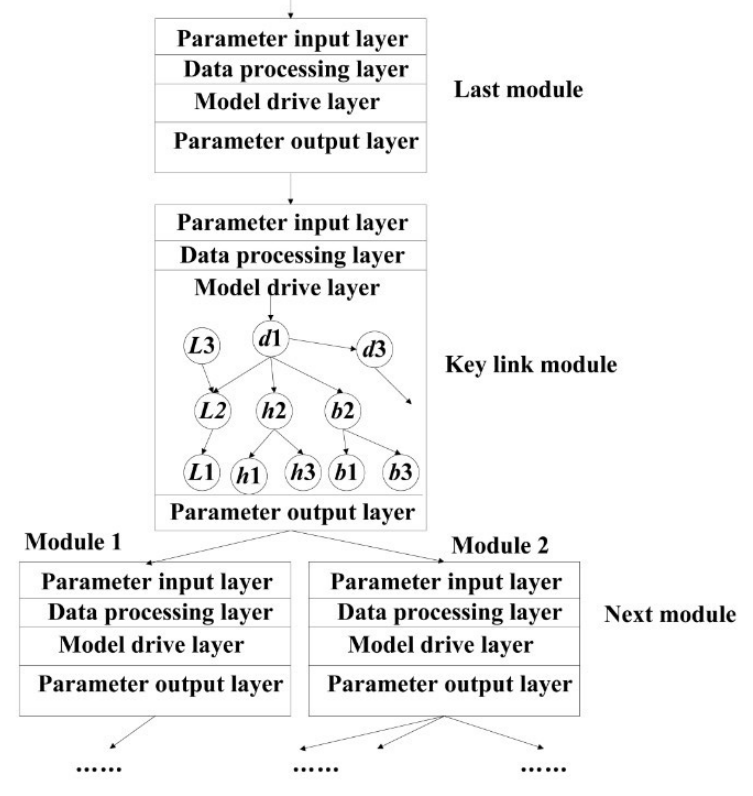

Figure 6 Parameter transfer model

with each other.

\subsection{Parameter transfer model}

There exist steady association relations between parts in subassembly interior. So we put to use the idea of modularization design. Take each subassembly as a module. Each module has input parameters and output parameters. These parameters are the link between modules and they are also participants to fulfill parameter transfer. Input parameters of the module drive the correlative part of module interior to change. At the same time, changed parameter as output parameter is transferred to the next module. Parameter transfer model is as shown in Figure 6.

Model file of the module is divided into four layers, namely, parameter input layer, data processing layer, model drive layer and parameter output layer. Parameter input layer receives the parameters from the last module. It includes variant parameter of module itself and of all subassembly and parts. By data processing, some parameters drive part or subassembly itself to change. The others are separated and transferred to the next module as variant parameters.

\subsection{Dimension constraint network structure}

We discuss the network structure for dimension constraints relations. In the variant design process, different types of parts have different priority values when solving constraints that part dimensions should be satisfied. Various forms exist for parameter constraints of parts and transfer path, and a large and complex

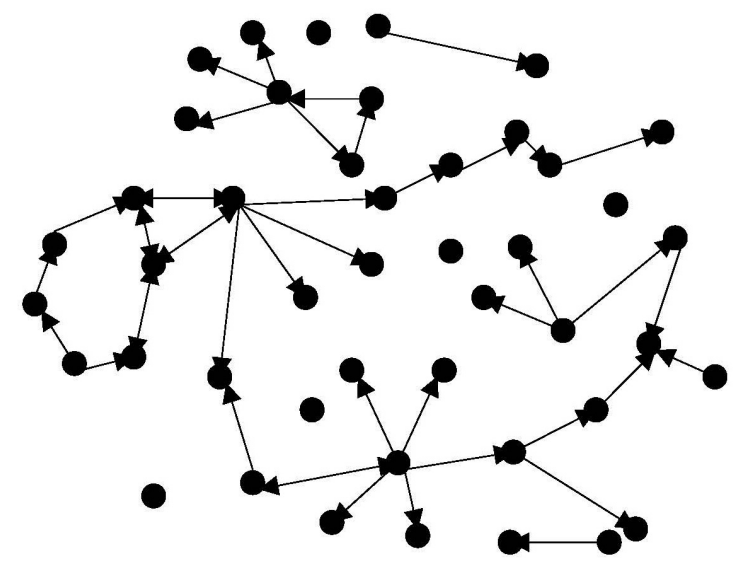

Figure 7 Network Structure for Dimension Constraints

network structure for dimension constraints relations will be formed among dimension constraints of all parts in a product design. Figure 7 shows a network structure in which nodes are used for presenting dimension parameters, and edges are used for presenting constraints relationships between dimension parameters. In the network structure all edges are directed, and the direction of edges starts from one node to the constrained node. When an edge is a two-way arrow, the pointed nodes (presenting dimension parameters) exist assembly relations and the two parameters are constrained each other.

Taking complexity of mechanical products into account, a complex network structure between the parts is formed, which is difficult to describe in a unified form. However, the network structure will become more complicated after distinguishing part types because transfer and assignment among dimensions constraints can not be done directly. Figure 8(a) shows a complete relation of dimensions constraints among parts; Figure 8(b) shows a relation of one dimension constraints multi-dimensions at the same time; Figure 8(c) shows a relation of multi-dimensions constraint one dimension; Figure 8(d) shows a relation of cycle constraints among parameters of parts; and Figure 8(e) shows a relation of one dimension does not have any constraints with others. The complex structure in product variant design will bring difficulty on part dimension parameter assignments which will increase complexity of the variant design process.

Therefore, in order to simplify the network structure of dimension constraints and to facilitate constraint solving, the method proposed in this paper is used in our study. Parts associated design based on constraint satisfaction priority and assembly relation- 


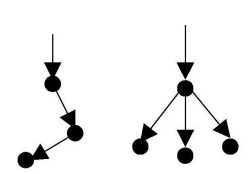

(a)

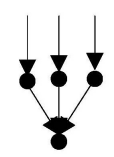

(c)

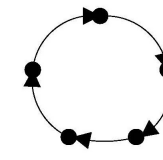

(d)

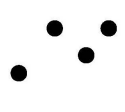

(e)
- $\quad$ Node ( presenting part dimension )

Constraint relations among dimension

Figure 8 Constraint forms between dimension parameter

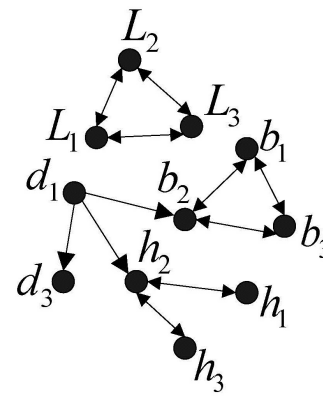

(a) Traditional network structure

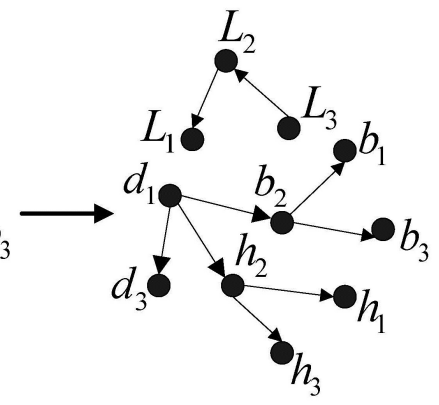

(b) Network structure based on constraint satisfaction priority
Figure 9 The reconstructed network structure for dimension constraints relations

ship is adopted, and the priority of parts is determined. So, parameters associated relations within the general assembly modules and among different modules, are built. And then, we can give a further simplify of the network structure which is similar to the structure of Figure 7. For example, Figure 9(a) shows a traditional network structure of the sub-module coupling. And Figure 9(a) can be simplified as Figure 9(b) by using idea of constraint satisfaction priority. In Figure 9(b), its priority from high to low is key, keyway shafts, and hub whole keyway; in accordance with the priority requirements, part of the key dimensions of parts is shown in Figure 9 (b).

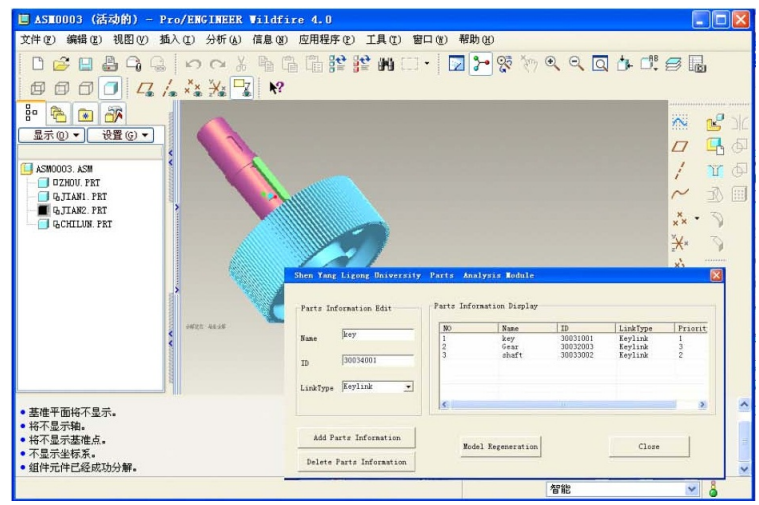

Figure 10 Constraint satisfaction priority definition of part

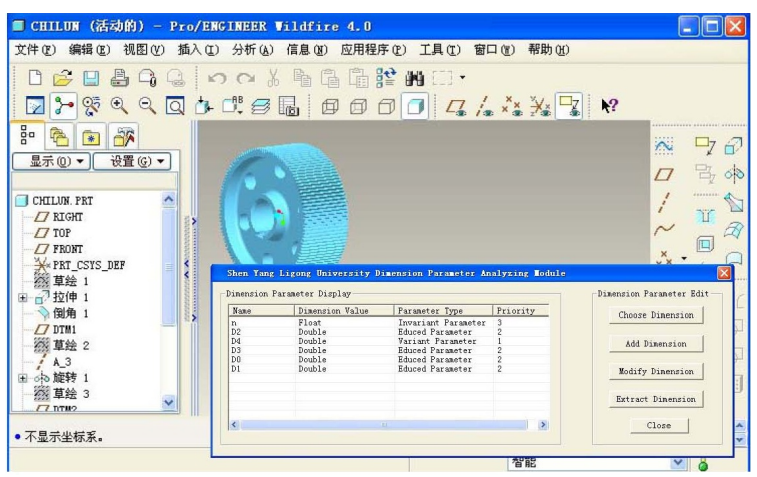

Figure 11 Dimension parameter analyzing

As can be seen from Figure 9, traditional network structure of dimensions constrained contains several bound forms of dimensions, including a dimension parameter constraints multiple dimensions and cycle constraints between parameters, which is not conducive to the transfer of dimension parameters. Network structure based on constraint satisfaction priority removes cycle constraints and ensures every edge has only one direction which makes the network simple and easy to solve the constraints.

\section{Case study}

The proposed method has been implemented. This section provides a case study based on the modeling method. As an example, Pro-E/ is taken as a 3D environment and $\mathrm{VC}++$ as a development tool; all methods are applied to a shaft-hub link of speed reducer product.

The developed modules of variant design mainly include the following functional modules: constraint satisfaction priority definition functional module of part; dimension parameter analyzing functional module; and dimension constraint relation management functional module.

In constraint satisfaction priority definition functional module, the main functions comprise part type define, constraint satisfaction priority value set. Its run interface is shown in Figure 10.

In dimension parameter analyzing functional module, the main functions contain add, delete, extract, type define of dimension parameter etc. The interface is shown in Figure 11.

In dimension constraint relation management functional module, the main functions contain add, delete, modify, extract of dimension constraint relation etc. Its run interface is shown in Figure 12. When parameter $\mathrm{d} 1$ of shaft is changed, the correlative parameters of shaft, key and gear are all changed too. The regen- 


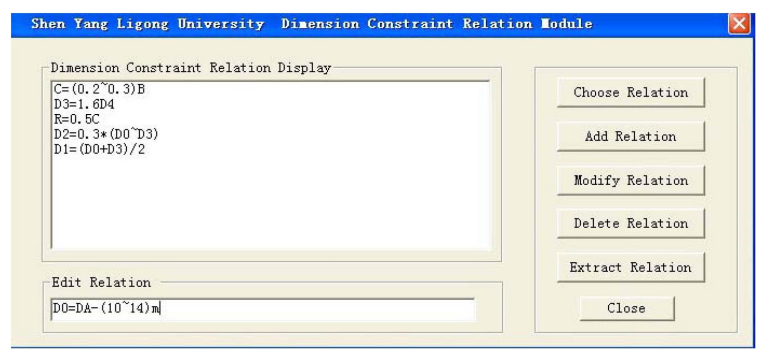

Figure 12 Dimension constraint relation edit interface

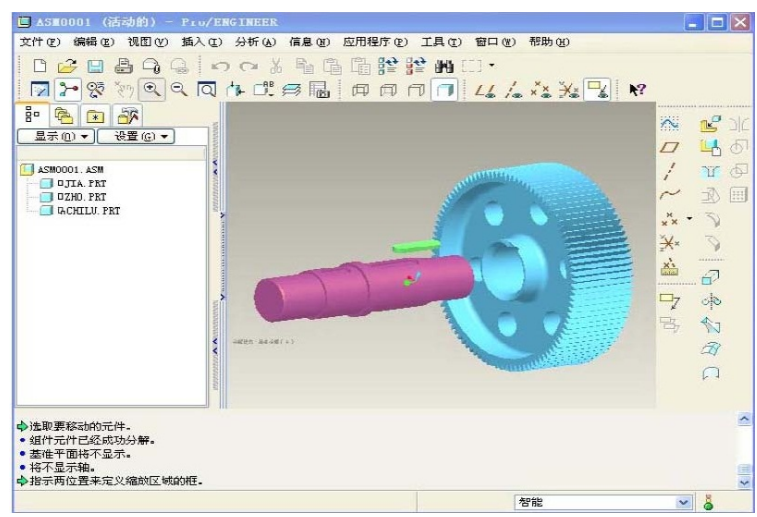

Figure 13 Regenerate model after dimension changed

erated 3D shaft-hub link model is illustrated in Figure 13.

\section{Conclusion}

This paper illustrates the various dimension parameter transfer considerations in the design of components and suggests methods for improved variant design. A detailed study of part and its dimension parameter classification and diversified assembly connections in variant product have been analyzed. According to part classification and its rate of reuse, different constraint satisfaction priority values for diverse parts have also been set. Moreover, constraint relations between parts based on assembly connection have been presented and parameter transfer structure model have been established. Various constraint forms between dimension parameters have been discussed through analyzing the variant parameters and variant relations of product and parts. The proposed parameter transfer method provides a new resolving for dimension modification and variant design. The further work studies on different algorithms for different parameter constraint forms. The methodology suggested has been validated through a case study by considering a typical shaft-hub structural components in speed reducer viz. took Pro-E as a $3 \mathrm{D}$ environment and $\mathrm{VC}++$ as a development tool.

\section{Acknowledgments}

The research is under the support of the National Natural Science Foundation of China with No. 50975183 and the National High-Tech. R\&D Program of China (No. 2009AA04Z167).

\section{References}

[1] B. Prasad, "Designing products for variety and how to managecomplexity", Journal of Product\&BrandManagement, Vol.7(3), pp.208-222, 1998.

[2] A. Michel, and V. Elise, "Configuration for mass customization: how to extend product configuration towards requirements and process configuration", Journal Intelligent Manufacturing, Vol.19, pp.521535 (Doi 10.1007/s10845-008-0135-z), 2008.

[3] J. X. Jiao, T. W. Simpson, Z. Siddique, "Product family design and platform-based product development: a state-of-the-art review", Journal Intelligent Manufacturing, Vol.18, pp.5-29 (Doi 10.1007/s10845-0070003-2), 2007.

[4] D. Battini, M. Faccio, E. Ferrari, A. Persona, F. Sgarbossa. "Design configuration for a mixed-model assembly system in case of low product demand", International Journal Advance Manufacturing Technology. Vol.34, pp.188-200, 2007.

[5] F. Y. Liu, X. L. Deng, “Dimension parameter modif ication and variant design method of conf iguration product", Computer Integrated Manufacturing Systems, vol.14, pp.2092-2096, Nov. 2008.

[6] S. L. Fang, Z. H. Sheng, "Parameter Transfer Structure for Complicated Product Variant Design", Computer Integrated Manufacturing Systems, vol.12, pp.1934-1938, Nov. 2006.

[7] S. L. Fang, Z. H. Shen. "Parameters transfer structure for complicated product variant design", Computer Integrated Manufacturing Systems, Vol.12, pp.19351938, Dec. 2006.

[8] X. L. Deng, F. Y. Liu and H. B. Wei. "Research on product dimension parameters analysis method and its application", Manufacturing Automation, 2009.

[9] Q. M. Wu, C. Zong, Q. Zhang, and W. Yang, "Research on Variant Design and Parameter Transfer Method for Complicated Products", Chinese Journal of Mechanical Engineering, vol.19, pp.2955-2959, Dec. 2008.

[10] Z.P.Wei. "Conjunction Design of Assembly of Rotary Shaft Based on Assembly Relation", Coal Mine Machinery, Vol.27, pp.74-76, Jan. 2006.

[11] X. L.Deng, F.Y.Liu. "Part Var iant Design Methods Ba sed on Parametr ic Technology", Machine Design and Research. Vol.24, pp.83-85, Aug. 2008. 
[12] Y. Li, Q. M. Wu, C. Zhou. "Representation of parameter association structure for generalized modular by directed graph", Computer Integrated Manufacturing System, Vol.15, pp.2312-2318, Dec.2009.

[13] J. Matthews, B. Singha, G. Mullineuxa and T. Medlanda. "Constraint-based approach to investigate the process flexibility of food processing equipment", Computers \& Industrial Engineering. Vol.51, pp. 809-820. Dec. 2006.

[14] S. W. Hsiao and E. Liu. "A structural componentbased approach for designing product family", Vol.56, pp.13-20. January 2005. 